\title{
The preventive analgesic efficacy of nefopam hydrochloride in acute and persistent pain after breast cancer surgery
}

\section{Jae-Hee Park, Bon-Wook Koo, Hyo-Suk Na, Ah-Young Oh \\ Department of Anesthesiology and Pain Medicine, Seoul National University Bundang Hospital, Seongnam, Korea}

Background: Breast cancer surgery is known to cause severe acute postoperative pain, which can persist for a long time. We administered nefopam preventively to patients undergoing total mastectomy with axillary lymph node dissection or sentinel lymph node biopsy, and evaluated its efficacy on acute and chronic postoperative pain.

Method: In this prospective randomised controlled study, patients were randomly allocated to one of two groups: nefopam or control. For the nefopam group $(n=21), 20 \mathrm{mg}$ of nefopam with normal saline $100 \mathrm{ml}$ was continuous infusion from before initiating the operation to the end of operation and the control group, normal saline $100 \mathrm{ml}$ was used. Meloxicam was prescribed in the postoperative period to all patients in both groups. Pain was assessed using a numerical rating scale (NRS), and postoperative chemotherapy, radiotherapy $(\mathrm{RT})$, or hormone therapy was evaluated.

Table1. Characteristics of Patient and Surgery

\begin{tabular}{lccc}
\hline & $\begin{array}{c}\text { Control } \\
(\mathbf{n}=\mathbf{2 2})\end{array}$ & $\begin{array}{c}\text { Nefopam } \\
(\mathbf{n}=\mathbf{2 1})\end{array}$ & P \\
\hline Age (years) & $61.1 \pm 13.8$ & $53.6 \pm 13.1$ & 0.075 \\
\hline Weight (kg) & $60.8 \pm 11$ & $57.5 \pm 9.1$ & 0.292 \\
\hline Height (cm) & $157.8 \pm 5.9$ & $155 \pm 6.2$ & 0.142 \\
\hline ASA (I / II) & $7 / 15$ & $11 / 10$ & 0.223 \\
\hline Total operation time (min) & $77.7 \pm 25.9$ & $76.7 \pm 30.6$ & 0.903 \\
\hline Total anesthesia time (min) & $100.5 \pm 29.6$ & $102.4 \pm 32.9$ & 0.841 \\
\hline Operation & & & 0.457 \\
\hline Total mastectomy & $19(86.4)$ & $16(76.2)$ & \\
\hline Modified radical mastectomy & $19(86.4)$ & $16(76.2)$ & \\
\hline Lymph node & & & 1.0 \\
\hline SLNB & $9(40.9)$ & $9(42.9)$ & \\
\hline ALND & $13(59.1)$ & $12(57.1)$ & \\
\hline
\end{tabular}

Values are meanstandard deviation or number (proportion). ALND = axillary lymph node dissection, SLNB = sentinel lymph node biopsy.
Result: The NRS of postoperative pain was significantly lower in the nefopam than in the control group at post operative $4 \mathrm{~h}(3.9 \pm 2.1$ vs $2.5 \pm 1.4, \mathrm{P}=$ 0.07 ) and in post anesthetic care unit reduced use of rescue analgesic drugs(35 \pm 22 vs $24.5 \pm 14.5, \mathrm{P}=$ 0.02) (table 2).

Conclusion: Preventive nefopam was helpful in reducing the acute postoperative pain, with reduced use of rescue analgesic drugs.

Table2. Postoperative variables

\begin{tabular}{|llll|}
\hline & $\begin{array}{l}\text { Control } \\
(\mathbf{n}=\mathbf{2 2})\end{array}$ & $\begin{array}{l}\text { Nefopam } \\
(\mathbf{n}=\mathbf{2 1})\end{array}$ & $\mathbf{P}$ \\
\hline $\begin{array}{l}\text { Total remifentanil }(\boldsymbol{\mu g}) \\
\text { Postoperative pain (NRS) }\end{array}$ & $317 \pm 211.6$ & $387.6 \pm 181.4$ & 0.138 \\
\hline PACU & $4.3 \pm 2.1$ & $4.2 \pm 1.8$ & 0.488 \\
\hline $\mathbf{4} \mathbf{~ h r}$ & $3.9 \pm 1.7$ & $2.5 \pm 1.4$ & 0.007 \\
\hline $\mathbf{2 4} \mathbf{~ h r}$ & $2.2 \pm 1.5$ & 1.80 .8 & 0.472 \\
\hline $\mathbf{4 8} \mathbf{~ h r}$ & $1.5 \pm 1.2$ & $1.1 \pm 0.9$ & 0.403 \\
\hline $\mathbf{7 2} \mathbf{~ h r}$ & $1.1 \pm 1.2$ & $0.7 \pm 0.9$ & 0.464 \\
\hline $\mathbf{1 0} \mathbf{~ d a y}$ & $0.6 \pm 0.7$ & $1 \pm 1.2$ & 0.295 \\
\hline $\mathbf{3} \mathbf{~ m o n t h}$ & $1 \pm 1.6$ & $1.3 \pm 1.7$ & 0.493 \\
\hline Fentanyl consumption $(\boldsymbol{\mu g})$ & & & \\
\hline PACU & $35 \pm 22$ & $24.5 \pm 14.5$ & 0.02 \\
\hline $\mathbf{4} \mathbf{~ h r}$ & $97.1 \pm 49.1$ & $87.5 \pm 32.6$ & 0.697 \\
\hline $\mathbf{2 4} \mathbf{~ h r}$ & $309.7 \pm 149.1$ & $285.2 \pm 139.5$ & 0.981 \\
\hline $\mathbf{4 8} \mathbf{~ h r}$ & $317.3 \pm 195.3$ & $276 \pm 221$ & 0.45 \\
\hline $\mathbf{7 2} \mathbf{~ h r}$ & $303 \pm 245$ & $190.9 \pm 216.4$ & 0.136 \\
\hline
\end{tabular}

Table3. Administration of Rescue Analgesic Drugs at PostOp Period

\begin{tabular}{|c|c|c|c|}
\hline & $\begin{array}{l}\text { Control } \\
(n=22)\end{array}$ & $\begin{array}{l}\text { Nefopam } \\
(n=21)\end{array}$ & $\mathbf{P}$ \\
\hline \multicolumn{4}{|c|}{ Postoperative } \\
\hline $4 \mathrm{hr}$ & $2(9.1)$ & $2(9.5)$ & 1.0 \\
\hline $24 \mathrm{hr}$ & $1(4.5)$ & $0(0)$ & 1.0 \\
\hline $48 \mathrm{hr}$ & $1(4.5)$ & $1(4.8)$ & 1.0 \\
\hline $72 \mathrm{hr}$ & $2(9.1)$ & $3(14.3)$ & 0.664 \\
\hline
\end{tabular}

Table4. Number of Patients Who Complained of Postoperative Pain

\begin{tabular}{|c|c|c|c|c|c|c|}
\hline \multicolumn{3}{|c|}{ Control $(n=22)$} & \multicolumn{3}{|c|}{ Nefopam $(n=21)$} & \\
\hline Postop 3 mo & \multicolumn{2}{|c|}{$11(50)$} & \multicolumn{2}{|c|}{$12(57.1)$} & 1.0 & \multirow{2}{*}{$\begin{array}{l}\text { Data were presented as number } \\
\text { (proportion). P1=comparison in } \mathrm{RT} \\
\text { subgroups, } \mathrm{P} 2=\text { comparison in non-RT } \\
\text { subgroups }\end{array}$} \\
\hline & $7(31.8)$ & $15(68.2)$ & $6(28.6)$ & $15(71.4)$ & P1:1.0 P2:1.0 & \\
\hline
\end{tabular}

